Sitzungsber. Abt. II (2005) 214: 141-160 Sitzungsberichte

Mathematisch-naturwissenschaftliche Klasse Abt. II

Mathematische, Physikalische und Technische Wissenschaften

(c) Österreichische Akademie der Wissenschaften 2006

Printed in Austria

\title{
Orthogonale Polynomlösungen von Differenzengleichungen vierter Ordnung
}

\author{
Von \\ Peter A. Lesky \\ (Vorgelegt in der Sitzung der math.-nat. Klasse am 17. November 2005 \\ durch das w. M. Johann Cigler)
}

\begin{abstract}
For difference equations of fourth order orthogonal polynomial solutions are given. A finite special case is completely treated.

Mathematics Subject Classification (2000): 33C45.

Key words: Orthogonal polynomials, difference equations of fourth order.
\end{abstract}

Nach den Untersuchungen von Differentialgleichungen vierter Ordnung auf (positiv definite) orthogonale Polynomlösungen durch H. L. Krall [1, 2], A. M. Krall [3, 4], Everitt [5] und LesKy $[6,7]$ ist es naheliegend, auch Differenzengleichungen vierter Ordnung auf (positiv definite) orthogonale Polynomlösungen zu untersuchen. In [1-7] sind folgende Polynomsysteme dargestellt: Hermite, Laguerre, Jacobi, Laguerretyp, Legendretyp, Jacobityp (unendliche Systeme) und Romanovski-Jacobi, Romanovski-Bessel, Romanovski-Pseudojacobi (endliche Systeme, [8]). Die Systeme Hermite, Laguerretyp, Legendretyp und Jacobityp zeichnen sich dadurch aus, dass sie über dreigliedrige Rekursionen für die Polynomkoeffizienten entstehen (Hermite und Laguerretyp als symmetrische Polynome). Hier erfolgt eine entsprechende Untersuchung 
von Differenzengleichungen vierter Ordnung über dreigliedrige Rekursionen für die Polynomkoeffizienten.

\section{Polynomlösungen von linearen homogenen Differentialgleichungen vierter Ordnung}

Für die Polynomlösungen $y_{n}(x)$ vom $n$-ten Grad in $x(n=0,1,2, \ldots)$ der Differentialgleichung

$$
P_{4}(x) y_{n}^{(I V)}(x)+P_{3}(x) y_{n}^{\prime \prime \prime}(x)+P_{2}(x) y_{n}^{\prime \prime}(x)+P_{1}(x) y_{n}^{\prime}(x)=\lambda_{n} y_{n}(x)
$$

zeigt der Ansatz

$$
y_{n}(x)=\sum_{k=0}^{n} a_{n, k} \frac{x^{k}}{k !} \quad\left(n=0,1,2, \ldots ; a_{n, n} \neq 0\right),
$$

dass für die Koeffizienten von (1.1) nur Polynome

$$
\begin{aligned}
& P_{4}(x)=e_{4,0}+e_{4,1} x+e_{4,2} x^{2}+e_{4,3} x^{3}+e_{4,4} x^{4}, \\
& P_{3}(x)=e_{3,0}+e_{3,1} x+e_{3,2} x^{2}+e_{3,3} x^{3}, \\
& P_{2}(x)=e_{2,0}+e_{2,1} x+e_{2,2} x^{2} \\
& P_{1}(x)=e_{1,0}+e_{1,1} x
\end{aligned}
$$

in Frage kommen. Nach dem Einsetzen von (1.2) in (1.1) liefert der Koeffizientenvergleich bei $x^{n} / n$ ! die Eigenwerte

$$
\begin{aligned}
\lambda_{n}= & n\left\{e_{1,1}+(n-1) e_{2,2}+(n-1)(n-2) e_{3,3}\right. \\
& \left.+(n-1)(n-2)(n-3) e_{4,4}\right\} \quad(n=0,1,2, \ldots) .
\end{aligned}
$$

Der Koeffizientenvergleich bei $x^{k} / k$ ! liefert

$$
\begin{aligned}
(k- & n) e_{1,1} a_{n, k}+e_{1,0} a_{n, k+1}+(k-n)(k+n-1) e_{2,2} a_{n, k}+k e_{2,1} a_{n, k+1} \\
& +e_{2,0} a_{n, k+2}+(k-n)\left[k^{2}+k n+n^{2}-3(k+n)+2\right] e_{3,3} a_{n, k} \\
& +k(k-1) e_{3,2} a_{n, k+1}+k e_{3,1} a_{n, k+2}+e_{3,0} a_{n, k+3} \\
& +(k-n)\left[k^{3}+k^{2} n+k n^{2}+n^{3}-6\left(k^{2}+k n+n^{2}\right)+11(k+n)-6\right] \\
& +e_{4,4} a_{n, k}+k(k-1)(k-2) e_{4,3} a_{n, k+1}+k(k-1) e_{4,2} a_{n, k+2} \\
& +k e_{4,1} a_{n, k+3}+e_{4,0} a_{n, k+4}=0 .
\end{aligned}
$$


Zur Erhöhung der Übersichtlichkeit wird (1.5) noch folgendermaßen umgeordnet:

$$
\begin{aligned}
& a_{n, k}(k-n)\left\{e_{1,1}+(k+n-1) e_{2,2}+\left[k^{2}+k n+n^{2}-3(k+n)+2\right] e_{3,3}\right. \\
& \left.\quad+\left[k^{3}+k^{2} n+k n^{2}+n^{3}-6\left(k^{2}+k n+n^{2}\right)+11(k+n)-6\right] e_{4,4}\right\} \\
& \quad+a_{n, k+1}\left\{e_{1,0}+k e_{2,1}+k(k-1) e_{3,2}+k(k-1)(k-2) e_{4,3}\right\} \\
& \quad+a_{n, k+2}\left\{e_{2,0}+k e_{3,1}+k(k-1) e_{4,2}\right\}+a_{n, k+3}\left\{e_{3,0}+k e_{4,1}\right\} \\
& \quad+a_{n, k+4} e_{4,0}=0 \\
& \left(k=n, n-1, \ldots, 1,0 ; a_{n, n} \neq 0, a_{n, n+1}=a_{n, n+2}=a_{n, n+3}=a_{n, n+4}=0\right) .
\end{aligned}
$$

Die folgenden vier Fälle entstehen mit der Dreigliedrigkeit von (1.6):

\section{Hermite:}

$$
\begin{aligned}
& e_{4,0}=1, e_{4,1}=e_{4,2}=e_{4,3}=e_{4,4}=0, e_{3,1}=2 \varepsilon(\varepsilon \in \mathbb{R} \backslash\{0\}), \\
& e_{3,0}=e_{3,2}=e_{3,3}=0, e_{2,0}=2 \varepsilon \tau(\tau \in \mathbb{R}), e_{2,2}=4 \varepsilon^{2}, e_{2,1}=0, \\
& e_{1,1}=4 \varepsilon^{2}(\tau-1), e_{1,0} \quad \text { (symmetrisch). }
\end{aligned}
$$

\section{Laguerretyp:}

$$
\begin{aligned}
& e_{4,2}=1 ; e_{4,0}=e_{4,1}=e_{4,3}=e_{4,4}=0 ; e_{3,1}=4, e_{3,2}=2 ; \\
& e_{3,0}=e_{3,3}=0 ; e_{2,1}=v+4(v \in \mathbb{R}), e_{2,2}=1 ; e_{2,0}=0 ; \\
& e_{1,0}=v-2, e_{1,1}=v .
\end{aligned}
$$

\section{Legendretyp:}

$$
\begin{aligned}
& e_{4,0}=1, e_{4,2}=-2, e_{4,4}=1 ; e_{4,1}=e_{4,3}=0 ; e_{3,1}=-8, e_{3,3}=8 ; \\
& e_{3,0}=e_{3,2}=0 ; e_{2,0}=-4(t+3)(t \in \mathbb{R}), e_{2,2}=4(t+3) ; e_{2,1}=0 ; \\
& e_{1,1}=8 t ; e_{1,0}=0 \quad \text { (symmetrisch). }
\end{aligned}
$$

\section{Jacobityp:}

$$
\begin{aligned}
& e_{4,2}=1, e_{4,3}=-2, e_{4,4}=1 ; e_{4,0}=e_{4,1}=0 ; \\
& e_{3,1}=4, e_{3,2}=-2(r+4)(r \in \mathbb{R}), e_{3,3}=2(r+2) ; e_{3,0}=0 ; \\
& e_{2,1}=-r(f+4)(f \in \mathbb{R}), e_{2,2}=r(r+f+3) ; e_{2,0}=0 ; \\
& e_{1,0}=r(2-f), e_{1,1}=r(r f-2) .
\end{aligned}
$$

Bei den Systemen Laguerre, Jacobi, Romanovski-Jacobi, RomanovskiBessel und Romanovski-Pseudojacobi bleiben in (1.6) fünfgliedrige Koeffizientenrekursionen bestehen. 


\section{Polynomlösungen von linearen homogenen Differenzengleichungen vierter Ordnung}

Für die Polynomlösungen $y_{n}(x)$ vom $n$-ten $\operatorname{Grad}$ in $x(n=0,1,2, \ldots)$ der Differenzengleichung ${ }^{1}$

$$
\begin{aligned}
& Q_{4}(x) \Delta^{4} y_{n}(x)+Q_{3}(x) \Delta^{3} y_{n}(x)+Q_{2}(x) \Delta^{2} y_{n}(x)+Q_{1}(x) \Delta y_{n}(x) \\
& \quad=\mu_{n} y_{n}(x+2)
\end{aligned}
$$

$\left(\Delta y_{n}(x)=y_{n}(x+1)-y_{n}(x) ; \Delta^{j} y_{n}(x)=\Delta\left(\Delta^{j-1} y_{n}(x)\right)\right)$ zeigt der Ansatz [8]

$$
y_{n}(x)=\sum_{k=0}^{n} b_{n, k}\left(\begin{array}{c}
x+k-2 \\
k
\end{array}\right) \quad\left(n=0,1,2, \ldots ; b_{n, n} \neq 0\right),
$$

dass für die Koeffizienten von (2.1) nur Polynome

$$
\begin{aligned}
& Q_{4}(x)=f_{4,0}+f_{4,1} x+f_{4,2} x^{2}+f_{4,3} x^{3}+f_{4,4} x^{4}, \\
& Q_{3}(x)=f_{3,0}+f_{3,1} x+f_{3,2} x^{2}+f_{3,3} x^{3}, \\
& Q_{2}(x)=f_{2,0}+f_{2,1} x+f_{2,2} x^{2}, \\
& Q_{1}(x)=f_{1,0}+f_{1,1} x
\end{aligned}
$$

in Frage kommen. Vor dem Einsetzen von (2.2) in (2.1) erfolgen für die Polynome (2.3) noch gewisse Vorbereitungen:

$$
\begin{aligned}
x\left(\begin{array}{c}
x+k-2 \\
k-r
\end{array}\right)= & (k-r+1)\left(\begin{array}{c}
x+k-2 \\
k-r+1
\end{array}\right)+(2-r)\left(\begin{array}{c}
x+k-2 \\
k-r
\end{array}\right) \\
x^{2}\left(\begin{array}{c}
x+k-2 \\
k-s
\end{array}\right)= & (k-s+2)(k-s+1)\left(\begin{array}{c}
x+k-2 \\
k-s+2
\end{array}\right) \\
& +(k-s+1)(5-2 s)\left(\begin{array}{c}
x+k-2 \\
k-s+1
\end{array}\right) \\
& +(s-2)^{2}\left(\begin{array}{c}
x+k-2 \\
k-s
\end{array}\right) \quad(s=2,3,4) ; \\
x^{3}\left(\begin{array}{c}
x+k-2 \\
k-t
\end{array}\right)= & (k-t+3)(k-t+2)(k-t+1)\left(\begin{array}{c}
x+k-2 \\
k-t+3
\end{array}\right) \\
& +3(k-t+2)(k-t+1)(3-t)\left(\begin{array}{c}
x+k-2 \\
k-t+2
\end{array}\right)+
\end{aligned}
$$

\footnotetext{
${ }^{1}$ Das „Störglied“ $\mu_{n} y_{n}(x+2)$ wird im Hinblick auf die Orthogonalität verwendet.
} 


$$
\begin{aligned}
& +(k-t+1)[3(3-t)(2-t)+1]\left(\begin{array}{c}
x+k-2 \\
k-t+1
\end{array}\right) \\
& +(2-t)^{3}\left(\begin{array}{c}
x+k-2 \\
k-t
\end{array}\right) \quad(t=3,4) ; \\
x^{4}\left(\begin{array}{c}
x+k-2 \\
k-4
\end{array}\right)= & k(k-1)(k-2)(k-3)\left(\begin{array}{c}
x+k-2 \\
k
\end{array}\right) \\
& -2(k-1)(k-2)(k-3)\left(\begin{array}{c}
x+k-2 \\
k-1
\end{array}\right) \\
& +7(k-2)(k-3)\left(\begin{array}{c}
x+k-2 \\
k-2
\end{array}\right) \\
& -15(k-3)\left(\begin{array}{c}
x+k-2 \\
k-3
\end{array}\right)+16\left(\begin{array}{c}
x+k-2 \\
k-4
\end{array}\right) .
\end{aligned}
$$

Ferner beachte man $y_{n}(x+2)=\Delta^{2} y_{n}(x)+2 \Delta y_{n}(x)+y_{n}(x)$, so dass mit dem Ansatz (2.2)

$$
\begin{aligned}
y_{n}(x+2)= & \sum_{k=2}^{n} b_{n, k}\left(\begin{array}{c}
x+k-2 \\
k-2
\end{array}\right)+2 \sum_{k=1}^{n} b_{n, k}\left(\begin{array}{c}
x+k-2 \\
k-1
\end{array}\right) \\
& +\sum_{k=0}^{n} b_{n, k}\left(\begin{array}{c}
x+k-2 \\
k
\end{array}\right)
\end{aligned}
$$

entsteht. Nach dem Einsetzen von (2.2) in (2.1) und Koeffizientenvergleich bei

$$
\left(\begin{array}{c}
x+n-2 \\
n
\end{array}\right)
$$

ergeben sich die Eigenwerte

$$
\begin{aligned}
\mu_{n}= & n\left[f_{1,1}+(n-1) f_{2,2}+(n-1)(n-2) f_{3,3}\right. \\
& \left.+(n-1)(n-2)(n-3) f_{4,4}\right] \quad(n=0,1,2, \ldots) .
\end{aligned}
$$

Zum Aufbau der (zunächst fünfgliedrigen) Rekursion werden die vier Summanden von (2.1) untersucht, wobei auch das Störglied Verwendung findet:

$$
\begin{aligned}
& {\left[f_{1,0}+f_{1,1} x\right] \sum_{k=1}^{n} b_{n, k}\left(\begin{array}{c}
x+k-2 \\
k-1
\end{array}\right)} \\
& \quad=\sum_{k=1}^{n} b_{n, k}\left(\begin{array}{c}
x+k-2 \\
k-1
\end{array}\right)\left[f_{1,0}+f_{1,1}\right]+\sum_{k=0}^{n} b_{n, k}\left(\begin{array}{c}
x+k-2 \\
k
\end{array}\right) k f_{1,1}
\end{aligned}
$$




$$
\begin{aligned}
& {\left[f_{2,0}+f_{2,1} x+f_{2,2} x^{2}\right] \sum_{k=2}^{n} b_{n, k}\left(\begin{array}{c}
x+k-2 \\
k-2
\end{array}\right)-\mu_{n}\left[\sum_{k=2}^{n} b_{n, k}\left(\begin{array}{c}
x+k-2 \\
k-2
\end{array}\right)\right.} \\
& \left.+2 \sum_{k=1}^{n} b_{n, k}\left(\begin{array}{c}
x+k-2 \\
k-1
\end{array}\right)+\sum_{k=0}^{n} b_{n, k}\left(\begin{array}{c}
x+k-2 \\
k
\end{array}\right)\right] \\
& =\sum_{k=2}^{n} b_{n, k}\left(\begin{array}{c}
x+k-2 \\
k-2
\end{array}\right)\left[f_{2,0}-\mu_{n}\right]+\sum_{k=1}^{n} b_{n, k}\left(\begin{array}{c}
x+k-2 \\
k-1
\end{array}\right)\left[(k-1) f_{2,1}\right. \\
& \left.+(k-1) f_{2,2}-2 \mu_{n}\right]+\sum_{k=0}^{n} b_{n, k}\left(\begin{array}{c}
x+k-2 \\
k
\end{array}\right)\left[k(k-1) f_{2,2}-\mu_{n}\right] \\
& {\left[f_{3,0}+f_{3,1} x+f_{3,2} x^{2}+f_{3,3} x^{3}\right] \sum_{k=3}^{n} b_{n, k}\left(\begin{array}{c}
x+k-2 \\
k-3
\end{array}\right)} \\
& =\sum_{k=3}^{n} b_{n, k}\left(\begin{array}{c}
x+k-2 \\
k-3
\end{array}\right)\left[f_{3,0}-f_{3,1}+f_{3,2}-f_{3,3}\right] \\
& +\sum_{k=2}^{n} b_{n, k}\left(\begin{array}{c}
x+k-2 \\
k-2
\end{array}\right)\left[(k-2) f_{3,1}-(k-2) f_{3,2}+(k-2) f_{3,3}\right] \\
& +\sum_{k=1}^{n} b_{n, k}\left(\begin{array}{c}
x+k-2 \\
k-1
\end{array}\right)(k-1)(k-2) f_{3,2} \\
& +\sum_{k=0}^{n} b_{n, k}\left(\begin{array}{c}
x+k-2 \\
k
\end{array}\right) k(k-1)(k-2) f_{3,3} \text {; } \\
& {\left[f_{4,0}+f_{4,1} x+f_{4,2} x^{2}+f_{4,3} x^{3}+f_{4,4} x^{4}\right] \sum_{k=4}^{n} b_{n, k}\left(\begin{array}{c}
x+k-2 \\
k-4
\end{array}\right)} \\
& =\sum_{k=4}^{n} b_{n, k}\left(\begin{array}{c}
x+k-2 \\
k-4
\end{array}\right)\left[f_{4,0}-2 f_{4,1}+4 f_{4,2}-8 f_{4,3}+16 f_{4,4}\right] \\
& +\sum_{k=3}^{n} b_{n, k}\left(\begin{array}{c}
x+k-2 \\
k-3
\end{array}\right)\left[(k-3) f_{4,1}-3(k-3) f_{4,2}\right. \\
& \left.+7(k-3) f_{4,3}-15(k-3) f_{4,4}\right] \\
& +\sum_{k=2}^{n} b_{n, k}\left(\begin{array}{c}
x+k-2 \\
k-2
\end{array}\right)\left[(k-2)(k-3) f_{4,2}-3(k-2)(k-3) f_{4,3}\right. \\
& \left.+7(k-2)(k-3) f_{4,4}\right]+
\end{aligned}
$$




$$
\begin{aligned}
& +\sum_{k=1}^{n} b_{n, k}\left(\begin{array}{c}
x+k-1 \\
k-2
\end{array}\right)\left[(k-1)(k-2)(k-3) f_{4,3}\right. \\
& \left.-2(k-1)(k-2)(k-3) f_{4,4}\right] \\
& +\sum_{k=0}^{n} b_{n, k}\left(\begin{array}{c}
x+k-2 \\
k
\end{array}\right) k(k-1)(k-2)(k-3) f_{4,4} .
\end{aligned}
$$

Jetzt ist erkennbar, dass man durch Ausschaltung der Glieder mit dem Faktor

$$
\left(\begin{array}{c}
x+k-2 \\
k-3
\end{array}\right) \quad \text { und } \quad\left(\begin{array}{c}
x+k-2 \\
k-4
\end{array}\right)
$$

zu einer dreigliedrigen Rekursion gelangen kann. Diese Ausschaltung gelingt durch Erfüllung von

$$
\begin{aligned}
& f_{3,0}=f_{3,1}-f_{3,2}+f_{3,3} ; \\
& f_{4,0}=2 f_{4,1}-4 f_{4,2}+8 f_{4,3}-16 f_{4,4} ; \\
& f_{4,1}=3 f_{4,2}-7 f_{4,3}+15 f_{4,4} .
\end{aligned}
$$

Außerdem ist noch eine Umformung der verbleibenden Binomialkoeffizienten nötig. Dazu berechnet man

$$
\begin{aligned}
& \left(\begin{array}{c}
x+k-2 \\
k-1
\end{array}\right)=\left(\begin{array}{c}
x+k-1 \\
k-1
\end{array}\right)-\left(\begin{array}{c}
x+k-2 \\
k-2
\end{array}\right) ; \\
& \left(\begin{array}{c}
x+k-2 \\
k
\end{array}\right)=\left(\begin{array}{c}
x+k \\
k
\end{array}\right)-2\left(\begin{array}{c}
x+k-1 \\
k-1
\end{array}\right)+\left(\begin{array}{c}
x+k-2 \\
k-2
\end{array}\right)
\end{aligned}
$$

und erhält aus (2.7)

$$
\begin{aligned}
& {\left[f_{1,0}+\right.}\left.f_{1,1} x\right] \sum_{k=1}^{n} b_{n, k}\left(\begin{array}{c}
x+k-2 \\
k-1
\end{array}\right) \\
&= \sum_{k=0}^{n} b_{n, k}\left(\begin{array}{c}
x+k \\
k
\end{array}\right) k f_{1,1}+\sum_{k=1}^{n} b_{n, k}\left(\begin{array}{c}
x+k-1 \\
k-1
\end{array}\right) \\
& \cdot\left[f_{1,0}+f_{1,1}-2 k f_{1,1}\right]+\sum_{k=2}^{n} b_{n, k}\left(\begin{array}{c}
x+k-2 \\
k-2
\end{array}\right)\left[-f_{1,0}-f_{1,1}+k f_{1,1}\right] ; \\
& {\left[f_{2,0}+f_{2,1} x+f_{2,2} x^{2}\right] \sum_{k=2}^{n} b_{n, k}\left(\begin{array}{c}
x+k-2 \\
k-2
\end{array}\right)-\mu_{n}\left[\sum_{k=2}^{n} b_{n, k}\left(\begin{array}{c}
x+k-2 \\
k-2
\end{array}\right)\right.} \\
&\left.\quad+2 \sum_{k=1}^{n} b_{n, k}\left(\begin{array}{c}
x+k-2 \\
k-1
\end{array}\right)+\sum_{k=0}^{n} b_{n, k}\left(\begin{array}{c}
x+k-2 \\
k
\end{array}\right)\right]=
\end{aligned}
$$




$$
\begin{aligned}
& =\sum_{k=0}^{n} b_{n, k}\left(\begin{array}{c}
x+k \\
k
\end{array}\right)\left[k(k-1) f_{2,2}-\mu_{n}\right] \\
& +\sum_{k=1}^{n} b_{n, k}\left(\begin{array}{c}
x+k-1 \\
k-1
\end{array}\right)\left[f_{2,1}-(2 k-1) f_{2,2}\right](k-1) \\
& +\sum_{k=2}^{n} b_{n, k}\left(\begin{array}{c}
x+k-2 \\
k-2
\end{array}\right)\left[f_{2,0}-f_{2,1}(k-1)+f_{2,2}(k-1)^{2}\right] \text {; } \\
& {\left[f_{3,0}+f_{3,1} x+f_{3,2} x^{2}+f_{3,3} x^{3}\right] \sum_{k=3}^{n} b_{n, k}\left(\begin{array}{c}
x+k-2 \\
k-3
\end{array}\right)} \\
& =\sum_{k=0}^{n} b_{n, k}\left(\begin{array}{c}
x+k \\
k
\end{array}\right) k(k-1)(k-2) f_{3,3} \\
& +\sum_{k=1}^{n} b_{n, k}\left(\begin{array}{c}
x+k-1 \\
k-1
\end{array}\right)\left[f_{3,2}-2 k f_{3,3}\right](k-1)(k-2) \\
& +\sum_{k=2}^{n} b_{n, k}\left(\begin{array}{c}
x+k-2 \\
k-2
\end{array}\right)\left[f_{3,1}-k f_{3,2}+\left(k^{2}-k+1\right) f_{3,3}\right](k-2) \text {; } \\
& {\left[f_{4,0}+f_{4,1} x+f_{4,2} x^{2}+f_{4,3} x^{3}+f_{4,4} x^{4}\right] \sum_{k=4}^{n} b_{n, k}\left(\begin{array}{c}
x+k-2 \\
k-4
\end{array}\right)} \\
& =\sum_{k=0}^{n} b_{n, k}\left(\begin{array}{c}
x+k \\
k
\end{array}\right) k(k-1)(k-2)(k-3) f_{4,4} \\
& +\sum_{k=1}^{n} b_{n, k}\left(\begin{array}{c}
x+k-1 \\
k-1
\end{array}\right)\left[f_{4,3}-2(k+1) f_{4,4}\right](k-1)(k-2)(k-3) \\
& +\sum_{k=2}^{n} b_{n, k}\left(\begin{array}{c}
x+k-2 \\
k-2
\end{array}\right)\left[f_{4,2}-(k+2) f_{4,3}\right. \\
& \left.+\left(k^{2}+k+5\right) f_{4,4}\right](k-2)(k-3) \text {. }
\end{aligned}
$$

\section{Nach dem Einsetzen von}

$$
y_{n}(x)=\sum_{k=0}^{n} b_{n, k}\left(\begin{array}{c}
x+k-2 \\
k
\end{array}\right)
$$


in die Differenzengleichung (2.1) entsteht insgesamt (man beachte die Erfüllung von (2.8))

$$
\begin{aligned}
\sum_{k=0}^{n} & b_{n, k}\left(\begin{array}{c}
x+k \\
k
\end{array}\right)\left\{k f_{1,1}+k(k-1) f_{2,2}+k(k-1)(k-2) f_{3,3}\right. \\
& \left.+k(k-1)(k-2)(k-3) f_{4,4}-\mu_{n}\right\} \\
& +\sum_{k=1}^{n} b_{n, k}\left(\begin{array}{c}
x+k-1 \\
k-1
\end{array}\right)\left\{f_{1,0}+f_{1,1}-2 k f_{1,1}+(k-1) f_{2,1}\right. \\
& -(k-1)(2 k-1) f_{2,2}+(k-1)(k-2) f_{3,2}-2 k(k-1)(k-2) f_{3,3} \\
& \left.+(k-1)(k-2)(k-3) f_{4,3}-2(k-1)(k-2)(k-3)(k+1) f_{4,4}\right\} \\
& +\sum_{k=2}^{n} b_{n, k}\left(\begin{array}{c}
x+k-2 \\
k-2
\end{array}\right)\left\{-f_{1,0}-f_{1,1}+k f_{1,1}+f_{2,0}-(k-1) f_{2,1}\right. \\
& +(k-1)^{2} f_{2,2}+(k-2)^{2} f_{3,1}-k(k-2) f_{3,2}+\left(k^{2}-k+1\right)(k-2) f_{3,3} \\
& +(k-2)(k-3) f_{4,2}-(k-2)(k-3)(k+2) f_{4,3} \\
& \left.+\left(k^{2}+k+5\right)(k-2)(k-3) f_{4,3}\right\}=0 .
\end{aligned}
$$

Der Koeffizientenvergleich bei

$$
\left(\begin{array}{c}
x+k \\
k
\end{array}\right)
$$

liefert eine dreigliedrige Rekursion für die $b_{n, k}$ :

$$
\begin{aligned}
b_{n, k} & \left\{k\left[f_{1,1}+(k-1) f_{2,2}+(k-1)(k-2) f_{3,3}+(k-1)(k-2)(k-3) f_{4,4}\right]\right. \\
& +n\left[f_{1,1}+(n-1) f_{2,2}+(n-1)(n-2) f_{3,3}\right. \\
& \left.\left.+(n-1)(n-2)(n-3) f_{4,4}\right]\right\}+b_{n, k+1}\left\{f_{1,0}+f_{1,1}-2(k+1) f_{1,1}\right. \\
& +k f_{2,1}-k(2 k+1) f_{2,2}+(k-1) k f_{3,2}-2(k-1) k(k+1) f_{3,3} \\
& \left.+(k-2)(k-1) k f_{4,3}-2(k-2)(k-1) k(k+2) f_{4,4}\right\} \\
& +b_{n, k+2}\left\{-f_{1,0}-f_{1,1}+(k+2) f_{1,1}+f_{2,0}-(k+1) f_{2,1}\right. \\
& +(k+1)^{2} f_{2,2}+k^{2} f_{3,1}-k(k+2) f_{3,2}+\left(k^{2}+3 k+3\right) k f_{3,3} \\
& +(k-1) k f_{4,2}-(k-1) k(k+4) f_{4,3} \\
& \left.+\left(k^{2}+5 k+11\right)(k-1) k f_{4,4}\right\}=0 \\
(k & \left.=n, n-1, \ldots, 1,0 ; b_{n, n} \neq 0, b_{n, n+1}=b_{n, n+2}=0\right) .
\end{aligned}
$$




\section{Orthogonalität für Polynomlösungen von linearen homogenen Differenzengleichungen vierter Ordnung}

In der Differenzengleichung (2.1) setzt man zweckmäßig

$$
\begin{aligned}
& Q_{4}(x)=\sigma(x+4), \\
& Q_{3}(x)=\tau(x+3), \\
& Q_{2}(x)=\varphi(x+2), \\
& Q_{1}(x)=\psi(x+1)
\end{aligned}
$$

und multipliziert mit $w(x+2)$ :

$$
\begin{aligned}
w(x+2) & \sigma(x+4) \Delta^{4} y_{n}(x)+w(x+2) \tau(x+3) \Delta^{3} y_{n}(x) \\
& +w(x+2) \varphi(x+2) \Delta^{2} y_{n}(x)+w(x+2) \psi(x+1) \Delta y_{n}(x) \\
= & w(x+2) \mu_{n} y_{n}(x+2) \quad(n=0,1,2, \ldots) .
\end{aligned}
$$

Wird $w$ so bestimmt, dass die selbstadjungierte Form

$$
\begin{gathered}
\Delta^{2}\left\{w(x) \sigma(x+2) \Delta^{2} y_{n}(x)\right\}+\Delta\{[w(x+1) \varphi(x+1) \\
\left.\left.-\Delta^{2}(w(x-1) \sigma(x+1))\right] \Delta y_{n}(x+1)\right\} \\
=w(x+2) \mu_{n} y_{n}(x+2)
\end{gathered}
$$

aus (3.2) entsteht, dann kann damit die Orthogonalität der Polynomlösungen $y_{n}$ bezüglich der Gewichtsfunktion $w$ erreicht werden.

Zuerst bringt man die Differenzengleichungen (3.2) und (3.3) zur Übereinstimmung. Unter Verwendung der Produktregeln

$$
\begin{aligned}
\Delta[f(x) g(x)] & =f(x+1) \Delta g(x)+g(x) \Delta f(x), \\
\Delta^{2}[f(x) g(x)] & =f(x+2) \Delta^{2} g(x)+2(\Delta f(x+1)) \Delta g(x)+g(x) \Delta^{2} f(x)
\end{aligned}
$$

berechnen wir

$$
\begin{aligned}
\Delta^{2}\left\{w(x) \sigma(x+2) \Delta^{2} y_{n}(x)\right\}= & w(x+2) \sigma(x+4) \Delta^{4} y_{n}(x) \\
& +2(\Delta[w(x+1) \sigma(x+3)]) \Delta^{3} y_{n}(x) \\
& +\left(\Delta^{2}[w(x) \sigma(x+2)]\right) \Delta^{2} y_{n}(x),
\end{aligned}
$$


und mit Verwendung von $\Delta y_{n}(x+1)=\Delta^{2} y_{n}(x)+\Delta y_{n}(x)$ entsteht

$$
\begin{aligned}
\Delta\{[w(x & \left.\left.+1) \varphi(x+1)-\Delta^{2}(w(x-1) \sigma(x+1))\right]\left[\Delta^{2} y_{n}(x)+\Delta y_{n}(x)\right]\right\} \\
= & {\left[w(x+2) \varphi(x+2)-\Delta^{2}(w(x) \sigma(x+2))\right]\left[\Delta^{3} y_{n}(x)+\Delta^{2} y_{n}(x)\right] } \\
& +\left(\Delta\left[w(x+1) \varphi(x+1)-\Delta^{2}(w(x-1) \sigma(x+1))\right]\right)\left[\Delta^{2} y_{n}(x)\right. \\
& \left.+\Delta y_{n}(x)\right] \\
= & {\left[w(x+2) \varphi(x+2)-\Delta^{2}(w(x) \sigma(x+2))\right] \Delta^{3} y_{n}(x) } \\
& +\left\{w(x+2) \varphi(x+2)-\Delta^{2}(w(x) \sigma(x+2))\right. \\
& \left.+\Delta\left[w(x+1) \varphi(x+1)-\Delta^{2}(w(x-1) \sigma(x+1))\right]\right\} \Delta^{2} y_{n}(x) \\
& +\left(\Delta\left[w(x+1) \varphi(x+1)-\Delta^{2}(w(x-1) \sigma(x+1))\right]\right) \Delta y_{n}(x) .
\end{aligned}
$$

Beide Ergebnisse werden zusammengefasst:

$$
\begin{aligned}
\Delta^{2}\left\{w(x) \sigma(x+2) \Delta^{2} y_{n}(x)\right\}+\Delta\{[w(x+1) \varphi(x+1) \\
\left.\left.\quad-\Delta^{2}(w(x-1) \sigma(x+1))\right] \Delta y_{n}(x+1)\right\} \\
=w(x+2) \sigma(x+4) \Delta^{4} y_{n}(x)+[w(x+2) \varphi(x+2) \\
\quad+w(x+2) \sigma(x+4)-w(x) \sigma(x+2)] \Delta^{3} y_{n}(x) \\
\quad+[2 w(x+2) \varphi(x+2)-w(x+1) \varphi(x+1)-w(x+2) \sigma(x+4) \\
\quad+3 w(x+1) \sigma(x+3)-3 w(x) \sigma(x+2) \\
\quad+w(x-1) \sigma(x+1)] \Delta^{2} y_{n}(x)+[w(x+2) \varphi(x+2) \\
\quad-w(x+1) \varphi(x+1)-w(x+2) \sigma(x+4)-3 w(x+1) \sigma(x+3) \\
\quad+3 w(x) \sigma(x+2)+w(x-1) \sigma(x+1)] \Delta y_{n}(x) .
\end{aligned}
$$

Der Vergleich mit der Differenzengleichung (3.2) führt bei $\Delta^{3} y_{n}(x)$ auf

$$
w(x+2)[\varphi(x+2)+\sigma(x+4)-\tau(x+3)]=w(x) \sigma(x+2),
$$

während der Vergleich bei $\Delta^{2} y_{n}(x)$ auf

$$
\Delta[w(x+1) \varphi(x+1)]-\Delta^{3}[w(x-1) \sigma(x+1)]=0
$$

führt. Somit liefert der Vergleich bei $\Delta y_{n}(x)$

$$
w(x+2) \psi(x+1)=0 .
$$

An die Stelle der Pearsonschen Differentialgleichungen [6] treten die Differenzengleichungen (3.6), (3.7) und die Forderung $\psi(x+1)=0$ $\left(f_{1,0}=f_{1,1}=0\right)$. Wird $w$ aus (3.6) und (3.7) bestimmt, dann kann an die Stelle der Differenzengleichung (3.2) mit $\psi(x+1)=0$ deren selbstadjungierte Form (3.3) treten. 
Für die Orthogonalität der Polynomlösungen $y_{n}(x)$ bezüglich $w$ als Gewichtsfunktion multipliziert man die zu $n$ gehörende Differenzengleichung (3.3) mit $y_{m}(x+2)$ und die zu $m$ gehörende Differenzengleichung (3.3) mit $y_{n}(x+2)(n, m=0,1,2, \ldots)$ und erhält nach deren Subtraktion

$$
\begin{aligned}
&\left(\mu_{n}-\mu_{m}\right) w(x+2) y_{n}(x+2) y_{m}(x+2) \\
&=\left(\Delta^{2}\left\{w(x) \sigma(x+2) \Delta^{2} y_{n}(x)\right\}+\Delta\{[w(x+1) \varphi(x+1)\right. \\
&\left.\left.\left.-\Delta^{2}(w(x-1) \sigma(x+1))\right] \Delta y_{n}(x+1)\right\}\right) y_{m}(x+2) \\
&-\left(\Delta^{2}\left\{w(x) \sigma(x+2) \Delta^{2} y_{m}(x)\right\}+\Delta\{[w(x+1) \varphi(x+1)\right. \\
&\left.\left.\left.-\Delta^{2}(w(x-1) \sigma(x+1))\right] \Delta y_{m}(x+1)\right\}\right) y_{n}(x+2) .
\end{aligned}
$$

Wendet man die partielle Summation

$$
\begin{gathered}
\sum_{x=A}^{B} f(x+1) \Delta g(x)=[f(x) g(x)]_{x=A}^{B+1}-\sum_{x=A}^{B} g(x) \Delta f(x) \\
\quad(N=B-A \in \mathbb{N} ; A \rightarrow-\infty \text { und } B \rightarrow \infty \text { moglich })
\end{gathered}
$$

und die Produktregel (3.4) auf Teile von (3.9) an, so entstehen

$$
\begin{aligned}
\sum_{x=A}^{B}\left(\Delta^{2}\right. & \left.\left\{w(x) \sigma(x+2) \Delta^{2} y_{n}(x)\right\}\right) y_{m}(x+2) \\
= & {\left[\left(\Delta\left\{w(x) \sigma(x+2) \Delta^{2} y_{n}(x)\right\}\right) y_{m}(x+1)\right]_{x=A}^{B+1} } \\
& -\sum_{x=A}^{B}\left(\Delta\left\{w(x) \sigma(x+2) \Delta^{2} y_{n}(x)\right\}\right) \Delta y_{m}(x+1) \\
= & {\left[\left(\Delta\left\{w(x) \sigma(x+2) \Delta^{2} y_{n}(x)\right\}\right) y_{m}(x+1)\right]_{x=A}^{B+1} } \\
& -\left[\left\{w(x) \sigma(x+2) \Delta^{2} y_{n}(x)\right\} \Delta y_{m}(x)\right]_{x=A}^{B+1} \\
& +\sum_{x=A}^{B}\left\{w(x) \sigma(x+2) \Delta^{2} y_{n}(x) \Delta^{2} y_{m}(x)\right\}
\end{aligned}
$$

und

$$
\begin{aligned}
\sum_{x=A}^{B}(\Delta\{ & {\left[w(x+1) \varphi(x+1)-\Delta^{2}(w(x-1) \sigma(x+1))\right] } \\
& \left.\left.\cdot \Delta y_{n}(x+1)\right\}\right) y_{m}(x+2) \\
= & {\left[\left\{\left[w(x+1) \varphi(x+1)-\Delta^{2}(w(x-1) \sigma(x+1))\right]\right.\right.} \\
& \left.\left.\cdot \Delta y_{n}(x+1)\right\} y_{m}(x+1)\right]_{x=A}^{B+1}-\sum_{x=A}^{B}\{[w(x+1) \varphi(x+1) \\
& \left.\left.-\Delta^{2}(w(x-1) \sigma(x+1))\right] \Delta y_{n}(x+1)\right\} \Delta y_{m}(x+1) .
\end{aligned}
$$


Hier wird klar, warum im Störglied der Differenzengleichung (2.1) $y_{n}(x+2)$ verwendet wurde: Zieht man entsprechend (3.9) noch die mit $m$ und $n$ vertauschten Teile ab und summiert von $A$ bis $B$, dann entfallen bezüglich (3.11) die Summen

$$
\sum_{x=A}^{B}\left\{w(x) \sigma(x+2) \Delta^{2} y_{n}(x) \Delta^{2} y_{m}(x)\right\}
$$

und bezüglich (3.12) die Summen

$\sum_{x=A}^{B}\left\{\left[w(x+1) \varphi(x+1)-\Delta^{2}(w(x-1) \sigma(x+1))\right] \Delta y_{n}(x+1)\right\} \Delta y_{m}(x+1)$.

Somit ergibt sich schließlich

$$
\begin{aligned}
\left(\mu_{n}-\right. & \left.\mu_{m}\right) \sum_{x=A}^{B} w(x+2) y_{n}(x+2) y_{m}(x+2) \\
= & {\left[\left(\Delta\left\{w(x) \sigma(x+2) \Delta^{2} y_{n}(x)\right\}\right) y_{m}(x+1)\right.} \\
& -\left\{w(x) \sigma(x+2) \Delta^{2} y_{n}(x)\right\} \Delta y_{m}(x)+(\{w(x+1) \varphi(x+1) \\
& \left.\left.\left.-\Delta^{2}(w(x-1) \sigma(x+1))\right\} \Delta y_{n}(x+1)\right) y_{m}(x+1)\right]_{x=A}^{B+1}
\end{aligned}
$$

oder noch in einer anderen Form

$$
\begin{aligned}
\left(\mu_{n}-\right. & \left.\mu_{m}\right) \sum_{x=A}^{B} w(x+2) y_{n}(x+2) y_{m}(x+2) \\
= & {\left[\{w(x+1) \varphi(x+1)-w(x-1) \sigma(x+1)\}\left(\Delta y_{n}(x+1)\right) y_{m}(x+1)\right.} \\
& +w(x) \sigma(x+2)\left\{2\left[\Delta y_{n}(x)\right] y_{m}(x+1)+\left[\Delta^{2} y_{n}(x)\right] y_{m}(x)\right\} \\
& \left.+w(x+1) \sigma(x+3)\left[\Delta^{3} y_{n}(x)-\Delta y_{n}(x+1)\right] y_{m}(x+1)\right]_{x=A}^{B+1} .
\end{aligned}
$$

Liegt die Verschiedenheit der Eigenwerte $\mu_{n}$ vor, dann hat man Orthogonalität der Polynomlösungen $y_{n}(x+2)$ von (2.1) bezüglich $w(x+2)$, wenn rechts vom Gleichheitszeichen in (3.13) bzw. (3.14) für $n \neq m$ null entsteht (wegen $\psi(x+1)=f_{1,0}+f_{1,1} x$ gilt $\mu_{0}=\mu_{1}=0$, worauf bei der Orthogonalität geachtet werden muss). Das bedingt gewisse Eigenschaften von $\varphi$ und $\sigma$ in den „Randpunkten“ $A$ und $B$. Selbstverständlich sind auch $A \rightarrow-\infty$ und $B \rightarrow \infty$ möglich, wenn $w$ die erforderlichen Konvergenzeigenschaften erzeugt. 
Zur Erreichung der Orthogonalität auf $\{A, A+1, \ldots, B-1, B\}$ müssen $\varphi(x+1), \sigma(x+1), \sigma(x+2)$ und $\sigma(x+3)$ so gewählt werden, dass einerseits

$$
\begin{aligned}
& w(x+1) \varphi(x+1)-w(x-1) \sigma(x+1), \\
& w(x) \sigma(x+2)
\end{aligned}
$$

und

$$
w(x+1) \sigma(x+3)
$$

für $x=A$ und $x=B+1 \quad(N=B-A \in \mathbb{N} ; A \rightarrow-\infty$ und $B \rightarrow \infty$ möglich) null sind, andererseits die beiden Differenzengleichungen (3.6) und (3.7) und $\psi(x+1)=0$ gelten.

\section{Behandlung eines endlichen Spezialfalles}

Es wird der einfachste Fall mit $w(x)=1$ und Orthogonalität auf $\{0,1, \ldots, N\}(A=0, B=N)$ behandelt. Zunächst wählt man $\sigma$ derart, dass $\sigma(x+3)$ und $\sigma(x+2)$ für $x=0$ und $x=N+1$ null werden:

$$
\begin{aligned}
\sigma(x+4)= & (x+1)(x+2)(x-N)(x-N+1) \\
= & x^{4}+2(2-N) x^{3}+\left(N^{2}-7 N+5\right) x^{2} \\
& +\left(3 N^{2}-7 N+2\right) x+2 N(N-1) ; \\
\sigma(x+3)= & x(x+1)(x-N-1)(x-N) \\
= & x^{4}-2 N x^{3}+\left(N^{2}-N-1\right) x^{2}+N(N+1) x ; \\
\sigma(x+2)= & (x-1) x(x-N-2)(x-N-1) \\
= & x^{4}-2(2+N) x^{3}+\left(N^{2}+5 N+5\right) x^{2}-x(N+1)(N+2) ; \\
\sigma(x+1)= & (x-2)(x-1)(x-N-3)(x-N-2) \\
= & x^{4}-2(4+N) x^{3}+\left(N^{2}+11 N+23\right) x^{2} \\
& -\left(3 N^{2}+19 N+28\right) x+2(N+2)(N+3) .
\end{aligned}
$$

Damit liegen die Koeffizienten von $Q_{4}(x)$ aus (2.3) fest:

$$
\begin{aligned}
& f_{4,0}=2 N(N-1), \\
& f_{4,1}=(N-2)(3 N-1), \\
& f_{4,2}=N^{2}-7 N+5, \\
& f_{4,3}=2(2-N), f_{4,4}=1 .
\end{aligned}
$$


Mit diesen Koeffizienten sind die zweite und dritte Bedingung aus (2.8) erfüllt:

$$
\begin{aligned}
f_{4,0}- & 2 f_{4,1}+4 f_{4,2}-8 f_{4,3}+16 f_{4,4} \\
= & 2 N^{2}-2 N-6 N^{2}+14 N-4+4 N^{2}-28 N \\
& +20-32+16 N+16 \\
& =0
\end{aligned}
$$

Im nächsten Schritt berechnen wir $\sigma(1)=2(N+2)(N+3)$ und $\sigma(N+2)=2(N-1) N$, setzen $\varphi(x+1)=\alpha x^{2}+\beta x+\gamma$ und haben $\varphi(1)=\gamma$ und $\varphi(N+2)=\alpha(N+1)^{2}+\beta(N+1)+\gamma$. Im Sinne von (3.15) müssen die Gleichungen

$$
\begin{aligned}
(\varphi(1)-\sigma(1) & =) & \gamma-2(N+2)(N+3) & =0, \\
(\varphi(N+2)-\sigma(N+2) & =) & \alpha(N+1)^{2}+\beta(N+1)+\gamma-2(N-1) N & =0
\end{aligned}
$$

gelten; daraus folgen $\gamma=2(N+2)(N+3)$ und $\alpha(N+1)+\beta=-12$.

Ferner muß die Differenzengleichung (3.7) erfüllt werden. Dazu berechnen wir $\Delta \varphi(x+1)=2 \alpha x+\alpha+\beta$ und $\Delta^{3} \sigma(x+1)=$ $12(2 x-N-1)$ und haben für $(3.7)$

$$
\left(\Delta \varphi(x+1)-\Delta^{3} \sigma(x+1)=\right) \quad(2 \alpha-24) x+\alpha+\beta+12(N+1)=0 .
$$

Das liefert $\alpha=12$ und $\beta=-12(N+2)$ (im Einklang mit $\alpha(N+1)$ $+\beta=-12$ ), so dass sich

$$
\varphi(x+1)=12 x^{2}-12(N+2) x+2(N+2)(N+3)
$$

und

$$
\varphi(x+2)=12 x^{2}-12 N x+2 N(N-1)
$$

ergeben. Damit liegen die Koeffizienten von $Q_{2}(x)$ aus (2.3) fest:

$$
\begin{gathered}
f_{2,0}=2 N(N-1), \quad f_{2,1}=-12 N, \\
f_{2,2}=12 .
\end{gathered}
$$

Es bleibt die Erfüllung der Differenzengleichung (3.6), wobei $\tau(x+3)$ festgelegt wird:

$$
\begin{aligned}
\tau(x+3) & =\varphi(x+2)+\sigma(x+4)-\sigma(x+2) \\
& =8 x^{3}-12(N-1) x^{2}+4\left(N^{2}-4 N+1\right) x+4 N(N-1) .
\end{aligned}
$$


Damit liegen die Koeffizienten von $Q_{3}(x)$ aus (2.3) fest:

$$
\begin{aligned}
& f_{3,0}=4 N(N-1), \\
& f_{3,1}=4\left(N^{2}-4 N+1\right), \\
& f_{3,2}=-12(N-1), \\
& f_{3,3}=8 .
\end{aligned}
$$

Mit diesen Koeffizienten ist die erste Bedingung aus (2.8) erfüllt:

$f_{3,0}-f_{3,1}+f_{3,2}-f_{3,3}=4 N^{2}-4 N-4 N^{2}+16 N-4-12 N+12-8$

$$
=0 \text {. }
$$

Wegen $\psi(x+1)=0$ liegen noch $f_{1,0}=f_{1,1}=0$ fest.

Damit liegt folgende Differenzengleichung vierter Ordnung vor:

$$
\begin{aligned}
& {\left[2 N(N-1)+(N-2)(3 N-1) x+\left(N^{2}-7 N+5\right) x^{2}-2(N-2) x^{3}\right.} \\
& \left.\quad+x^{4}\right] \Delta^{4} y_{n}(x)+\left[4 N(N-1)+4\left(N^{2}-4 N+1\right) x-12(N-1) x^{2}\right. \\
& \left.\quad+8 x^{3}\right] \Delta^{3} y_{n}(x)+\left[2 N(N-1)-12 N x+12 x^{2}\right] \Delta^{2} y_{n}(x) \\
& =\mu_{n} y_{n}(x+2)
\end{aligned}
$$

mit

$$
\mu_{n}=(n-1) n(n+1)(n+2) \quad(n=0,1,2, \ldots) .
$$

Die dreigliedrige Rekursion (2.11) erhält folgende Gestalt:

$$
\begin{aligned}
b_{n, k}\{ & (k-1) k(k+1)(k+2)-(n-1) n(n+1)(n+2)\} \\
& \quad-b_{n, k+1} 2 k(k+1)(k+2)(N+k+2) \\
& \quad+b_{n, k+2}(k+1)(k+2)(N+k+2)(N+k+3)=0 \\
(k= & \left.n, n-1, n-2, \ldots, 1,0 ; b_{n, n} \neq 0, b_{n, n+1}=b_{n, n+2}=0\right) .
\end{aligned}
$$

Zur Berechnung der $b_{n, k}$ ist noch folgende Umformung der Rekursion zweckmäßig:

$$
\begin{gathered}
(n-k)(n-k+1)[n(n+1)+(k-1)(k+2)] b_{n, k}+(k+1)(k+2) \\
\cdot(N+k+2)\left[2 k b_{n, k+1}-(N+k+3) b_{n, k+2}\right]=0
\end{gathered}
$$

$\left(k=n, n-1, n-2, \ldots, 1,0 ; b_{n, n} \neq 0, b_{n, n+1}=b_{n, n+2}=0\right)$. Man findet damit

und

$$
b_{n, n-1}=-\frac{N+n+1}{2} b_{n, n}
$$

$$
\begin{aligned}
b_{n, k}= & (-1)^{n-k} \frac{(n-1)(n-2) \cdots(k+1)(N+n+1)(N+n) \cdots(N+k+2)}{2(n-k) !(2 n-1)(2 n-2) \cdots(n+k+1)} \\
& \times b_{n, n}
\end{aligned}
$$


$(k=n-2, n-3, \ldots, 1,0)$, so dass folgende Lösungspolynome von (4.7) entstehen:

$y_{n}(x)$
$=\left\{\left(\begin{array}{c}x+n-2 \\ n\end{array}\right)+\sum_{j=1}^{n} \frac{(-1)^{j}\left(\begin{array}{c}N+n+1 \\ j\end{array}\right)\left(\begin{array}{c}n-1 \\ j-1\end{array}\right)}{2\left(\begin{array}{c}2 n-1 \\ j-1\end{array}\right)}\left(\begin{array}{c}x+n-2-j \\ n-j\end{array}\right)\right\} b_{n, n}$

$(n=1,2, \ldots)$ und $y_{0}(x)=1$; diese werden mit $b_{n, n}=n !$ monisch.

Wir geben die (monischen) Polynome $y_{n}(x)$ und $y_{n}(x+2)$ $(n=1,2,3,4)$ an:

$$
\begin{aligned}
y_{1}(x)= & x-\frac{N+4}{2}, \\
y_{1}(x+2)= & x-\frac{N}{2} ; \\
y_{2}(x)= & x^{2}-(N+4) x+\frac{(N+3)(N+8)}{6}, \\
y_{2}(x+2)= & x^{2}-N x+\frac{(N-1) N}{6} ; \\
y_{3}(x)= & x^{3}-\frac{3}{2}(N+4) x^{2}+\frac{1}{10}\left(6 N^{2}+57 N+122\right) x \\
& -\frac{1}{20}(N+3)(N+4)(N+14), \\
y_{3}(x+2)= & x^{3}-\frac{3}{2} N x^{2}+\frac{1}{10}\left(6 N^{2}-3 N+2\right) x-\frac{1}{20}\left(N^{3}-3 N^{2}+2 N\right) ; \\
y_{4}(x)= & x^{4}-2(N+4) x^{3}-\frac{1}{7}\left(9 N^{2}-81 N+173\right) N^{2} \\
& -\frac{1}{7}\left(2 N^{3}+33 N^{2}+161 N+244\right) x \\
& +\frac{1}{70}(N+3)(N+4)(N+5)(N+22), \\
& x^{4}-2 N x^{3}+\frac{1}{7}\left(9 N^{2}-3 N+5\right) x^{2}-\frac{N}{7}\left(2 N^{2}-3 N+5\right) x \\
& +\frac{N}{70}\left(N^{3}-6 N^{2}+11 N-6\right) . \\
y_{4}(x+2) &
\end{aligned}
$$


Man beachte, dass im konstanten Glied von $y_{n}(x+2)(n=1,2,3,4)$ nur Potenzen von $N$ vorkommen.

Die (monischen) Lösungspolynome (4.11) betrachten wir im Hinblick auf die Orthogonalität in der Form

$$
y_{n}(x+2)=\sum_{j=0}^{n} \alpha_{n, k}(x+2)^{k} \quad\left(n=0,1,2, \ldots ; \alpha_{n, n}=1\right) .
$$

Zur Berechnung der dreigliedrigen Rekursion ${ }^{2}$

$$
\begin{aligned}
y_{0}(x+2) & =1, \\
y_{1}(x+2) & =x+2-c_{0}, \\
y_{n+1}(x+2) & =\left(x+2-c_{n}\right) y_{n}(x+2)-d_{n} y_{n-1}(x+2) \quad(n=1,2,3, \ldots)
\end{aligned}
$$

setzt man (4.12) in (4.13) ein und erhält durch Koeffizientenvergleich bei $(x+2)^{n}$ und $(x+2)^{n-1}$

$c_{0}=-\alpha_{1,0}$

$c_{n}=\alpha_{n, n-1}-\alpha_{n+1, n}$

$d_{n}=\alpha_{n, n-2}-\alpha_{n+1, n-1}-c \alpha_{n, n-1} \quad\left(n=1,2,3, \ldots ; \alpha_{1,-1}=0\right)$.

Zur Aufstellung der Rekursion genügen also $\alpha_{n, n-1}$ und $\alpha_{n, n-2}$. In diesem Sinne werden die entsprechenden Koeffizienten aus (4.11) berechnet:

$$
\begin{gathered}
\left(\begin{array}{c}
x+n \\
n
\end{array}\right) n !=(x+n)(x+n-1) \cdots(x+1) \\
=x^{n}+\frac{n(n+1)}{2} x^{n-1}+\frac{n(n-1)(n+1)(3 n+2)}{24} x^{n-2}+\cdots ; \\
\sum_{j=1}^{n} \frac{(-1)^{j}\left(\begin{array}{c}
N+n+1 \\
j
\end{array}\right)\left(\begin{array}{c}
n-1 \\
j-1
\end{array}\right)}{2\left(\begin{array}{c}
2 n-1 \\
j-1
\end{array}\right)}\left(\begin{array}{c}
x+n-j \\
n-j
\end{array}\right) n ! \\
=-\frac{n(N+n+1)}{2} x^{n-1}-\frac{n^{2}(n-1)(N+n+1)}{4} x^{n-2}-\cdots \\
+\frac{n(n-1)^{2}(N+n)(N+n+1)}{4(2 n-1)} x^{n-2}+\cdots
\end{gathered}
$$

\footnotetext{
${ }^{2}$ Analog wie in [8] kann die Existenz einer dreigliedrigen Rekursion gezeigt werden.
} 
Damit entstehen

$\alpha_{n, n-1}=\frac{n(n+1)}{2}-\frac{n(N+n+1)}{2}=-\frac{n N}{2} \quad\left(n=1,2,3, \ldots ; \alpha_{1,1}=1\right)$

und

$$
\begin{aligned}
& c_{0}=-\alpha_{1,0}=\frac{N}{2}, \\
& c_{n}=\alpha_{n, n-1}-\alpha_{n+1, n}=-\frac{n N}{2}+\frac{(n+1) N}{2}=\frac{N}{2} \quad(n=1,2,3, \ldots) .
\end{aligned}
$$

Ferner ergibt sich

$$
\begin{array}{r}
\alpha_{n, n-2}=\frac{n(n-1)}{24(2 n-1)}\left\{6(n-1) N^{2}-6 N+(n-2)(n+1)\right\} \\
\left(n=1,2,3, \ldots ; \alpha_{1,-1}=0\right)
\end{array}
$$

zur Berechnung von

$$
\begin{aligned}
d_{n}= & \alpha_{n, n-2}-\alpha_{n+1, n-1}-c_{n} \alpha_{n, n-1} \\
= & -\frac{n}{4\left(4 n^{2}-1\right)}\left\{(n-1)^{2}(2 n+1) N^{2}-(n-1)(2 n+1) N\right. \\
& +\frac{1}{6}(n-2)\left(n^{2}-1\right)(2 n+1)-n(n+1)(2 n-1) N^{2} \\
& \left.+(n+1)(2 n-1) N-\frac{1}{6}\left(n^{2}-1\right)(n+2)(2 n-1)\right\}+\frac{n N^{2}}{4} \\
= & \frac{n}{4\left(4 n^{2}-1\right)}\left\{\left(-4 n^{2}+n+1\right) N^{2}+2 n N-n\left(n^{2}-1\right)\right\}+\frac{n N^{2}}{4} \\
= & -\frac{n N^{2}}{4}+\frac{n^{2}(N+1-n)(N+1+n)}{4}+\frac{n N^{2}}{4} \\
= & \frac{n^{2}(N+1-n)(N+1+n)}{4\left(4 n^{2}-1\right)} \quad(n=1,2,3, \ldots) .
\end{aligned}
$$

Nun wird die mit dem Satz von Favard zusammenhängende Theorie für positiv definite (endliche) Orthogonalsysteme herangezogen [8].

Man erkennt, dass die $d_{n}$ für $n=1,2, \ldots, N$ positiv sind, während $d_{N+1}=0$ ist, so dass nur ein endliches positiv definites Orthogonalsystem mit $N+1$ Polynomen entstehen kann. Für die (positiven) Normierungsfaktoren $\sigma_{n}=d_{0} d_{1} \cdots d_{n}$ mit

$$
d_{0}=\sum_{x=0}^{N} y_{0}(x)=N+1
$$


ergibt sich

$$
\begin{gathered}
\sigma_{n}=\left(\begin{array}{c}
N+1+n \\
2 n+1
\end{array}\right) \frac{(2 n+1) !}{4^{n}} \prod_{k=1}^{n} \frac{k^{2}}{4 k^{2}-1} \\
\left(n=1,2, \ldots, N ; \sigma_{0}=N+1\right) ;
\end{gathered}
$$

also hat man für die Polynomlösungen $y_{n}(x+2)$ von (4.7) die Orthogonalitätsrelation

$$
\begin{aligned}
\sum_{x=0}^{N} 1 \cdot y_{n}(x+2) \cdot y_{m}(x+2) & \text { für } n \neq m, \\
= & \begin{cases}0 & \text { für } n=m(\neq 0), \\
\left(\begin{array}{c}
N+1+n \\
2 n+1
\end{array}\right) \frac{(2 n+1) !}{4^{n}} \prod_{k=1}^{n} \frac{k^{2}}{4 k^{2}-1} & \text { für } n=m(=0) \\
(n, m=0,1, \ldots, N) . & (4.20\end{cases}
\end{aligned}
$$

\section{Literatur}

[1] KRALL, H. L. (1938) Certain differential equations for Tschebyscheff polynomials. Duke Math. J. 4: 705-718

[2] KRALL, H. L. (1940) On orthogonal polynomials satisfying a certain fourth order differential equation. The Pennsylv. State Coll. Bull. 34: 3-24

[3] KRALL, A. M. (1981) Orthogonal polynomials satisfying fourth order differential equations. Proc. Roy. Soc. Edinburgh, Sec. A 87: 271-288

[4] KRall, A. M. (2002) Hilbert Space, Boundary Value Problems and Orthogonal Polynomials. Birkhäuser, Basel-Boston-Berlin

[5] Everitt, W. N., LitTlejohn, L. L. (1988) Differential operators and the Legendre type polynomials. Diff. Int. Equations 1: 97-116

[6] LESKY, P. A. (1997) Eigenwertprobleme mit Differentialgleichungen vierter Ordnung für die kontinuierlichen klassischen Orthogonalpolynome. Sitzungsber. Öst. Akad. Wiss. Wien, math.-nat. Kl., Abt. II 206: 127-139

[7] LESKY, P. A. (1998) Eigenwertprobleme mit Differentialgleichungen vierter Ordnung für die Orthogonalpolynome vom Laguerretyp, Legendretyp und Jacobityp. Sitzungsber. Öst. Akad. Wiss. Wien, math.-nat. Kl., Abt. II 207: 23-34

[8] LESKY, P. A. (2005) Eine Charakterisierung der klassischen kontinuierlichen, diskreten und $q$-Orthogonalpolynome. Shaker, Aachen

Anschrift des Verfassers: Peter A. Lesky, G.-Hauptmann-Straße 4, 6020 Innsbruck, Österreich; Universität Stuttgart/Mathematik, Pfaffenwaldring 57, 70569 Stuttgart, Deutschland. E-Mail: pa.lesky@mathematik.uni-stuttgart.de. 